\title{
Impact de quelques techniques de lavage sur la décontamination de tuiles et béton contaminés par du cesium et du strontium radioactifs
}

\author{
C. CAMARASA-CLARET*, F. PERSIN**, J. REAL*
}

(Manuscrit reçu le 19 mars 1999, révisé le 9 juin 1999, accepté le 16 octobre 1999)

RESUMÉ Un accident sur un réacteur nucléaire avec dépôt de radionucléides sur des surfaces urbaines pourrait présenter un danger d'irradiation externe pour les populations. Afin d'appréhender ce risque, et compte tenu du peu de données disponibles dans la littérature, il nous est apparu opportun de tester en laboratoire l'efícacité de techniques de décontamination sur des surfaces urbaines judicieusement choisies avant d'envisager une étude de faisabilité à plus grande échelle. L'originalité de ce travail a consisté à évaluer, par le biais d'expérimentations en milieu contrôlé, l'efficacité d'une pluie et de lavages avec des solutions agressives sur des tuiles en argile et du béton contaminés avec des aérosols de césium et de strontium. L'eau de pluie s'est révélée peu efficace pour éliminer les radionucléides, son action est d'autant plus faible que l'intensité de la pluie est élevée. Une plus grande efficacité a été obtenue après 2 h 30 de lavage avec la solution $\mathrm{NH}_{4} \mathrm{Cl}\left(0,1 \mathrm{~mol} \mathrm{l}^{-1}\right)$ appliquée directement après la contamination. L'activité résiduelle est alors de $90 \%$ sur les tuiles et de $40 \%$ sur le béton. Les résultats obtenus sont moyens pour le béton mais peu satisfaisants pour les tuiles et confirment, dans des conditions de dépôt accidentel, la difficulté de déplacer une telle contamination.

ABSTRACT Washing processes impact on the decontamination of tiles and concrete contaminate by radioactive cesium and strontium.

An accident on a nuclear reactor with a radionuclide deposition on urban surfaces could present a danger of external of irradiation for the populace. In order to evaluate this risk, and taking into account the few available data in the literature, it appeared essential to us to test in the laboratory the efficacy of decontamination techniques on judiciously selected urban surfaces before considering a feasibility study on a large scale. The originality of this work consisted in evaluating by the means of experiments in controlled environment the decontaminating efficiency of a rain event and washings with aggressive solutions on clay tiles and concrete contaminated with strontium and cesium aerosols. The rainwater proved not to be very to be effective in eliminating the radionuclides, the weaker its effect the higher its intensity. A greater efficiency was obtained after $2 \mathrm{h30}$ of washing with the $\mathrm{NH}_{4} \mathrm{Cl}$ $0,1 \mathrm{~mol} \mathrm{l}^{-1}$ solution applied directly after the contamination. The residual activity then amounts to $90 \%$ on the tiles and $40 \%$ on the concrete. The results obtained are average for the concrete but not very satisfactory for the tiles and confirm, under conditions of accidental deposit, the difficulty in moving such a contamination.

\footnotetext{
* IPSN, Institut de protection et de sûreté nucléairc, département de protection de l'environnement, service d'études et de recherches, C.E. Cadarache, 13108 Saint-Paul-Lez-Durance Cedex, France.

** CNRS LMPM, UMR 5635, 1919, route de Mende, 34293 Montpellier Cedex 5, France.
} 


\section{Introduction}

Le devenir des radionucléides et l'évaluation des risques tout au long de leur transfert dans l'environnement font partie des éléments pris en compte pour la délivrance d'autorisations administratives pour les installations nucléaires ou l'élaboration des plans d'urgence en cas d'accident nucléaire.

Suite à l'accident de Tchernobyl, de nombreuses études ont été réalisées dans toute l'Europe pour évaluer la contamination et suivre son devenir. Certaines ont montré que les eaux de pluie n'induisent qu'une faible décontamination des matériaux urbains contaminés par du césium (Roed and Sandalls, 1989; Roed and Jacob, 1990; Nicholson and Hedgecock, 1991). Ces matériaux constituent donc des sources potentielles d'irradiation ou même de contamination par la poussière pour l'homme. De ce fait, un certain nombre d'essais ont été entrepris pour évaluer l'intérêt de la décontamination des surfaces urbaines. Il s'agit essentiellement de procédés mécaniques ou physico-chimiques. Parmi ces techniques, on peut citer notamment celles qui utilisent l'aspersion de solutions ammoniacales concentrées (Sandalls et al., 1986; De Witt et al., 1990 ; Roed and Anderson, 1996), mais aussi l'emploi de textiles échangeurs d'ions (Camarasa-Claret et al., 1997), de mousses (Gauchon et al., 1991) ou de polyacrylamides (Arnaud, 1995). Cependant la synthèse des résultats obtenus demeure très difficile compte tenu des conditions de mesure très variables, voire non précisées.

L'objectif de ce travail consiste donc à compléter des résultats mal connus et à tester la faisabilité technique de la méthode. Pour cela nous avons réalisé des expérimentations en milieu contrôlé afin d'évaluer l'efficacité du ruissellement de solutions d'eau de pluie ou de lavage sur le déplacement de césium et de strontium préalablement déposés sur des matériaux de construction. Le choix des matériaux a été guidé par l'importance de leur couverture des zones urbaines en France, et par leurs propriétés adsorbantes mises en évidence par les travaux effectués après l'accident de Tchernobyl (Devell, 1989; Nicholson, 1987; Roed and Sandalls 1989). Il s'agit de béton et de tuiles en argile. La contamination radioactive a été réalisée grâce à un dépôt d'aérosols radioactifs, selon un protocole simulant de façon réaliste les conditions accidentelles.

\section{Matériel et méthodes}

\subsection{Les matériaux}

Les matériaux de construction ont été prélevés à Marseille ; les tuiles «canal » appartenaient à une toiture construite vers 1910 et les plaques de béton à un trottoir datant des années 1970. Des analyses minéralogiques ont montré que les tuiles sont 
IMPACT DE QUELQUES TECHNIQUES DE LAVAGES...

\section{TABLEAU I}

Caractéristiques physiques des matériaux de construction étudiés. Physical characteristics of construction materials.

\begin{tabular}{|c|c|c|c|c|}
\hline Matériau & $\begin{array}{c}\text { Porosité totale } \\
\%\end{array}$ & $\begin{array}{c}\text { Diamètre médian } \\
\mu \mathrm{m}\end{array}$ & $\begin{array}{c}\text { Distribution des } \\
\text { pores } \mu \mathrm{m}\end{array}$ & $\begin{array}{c}\text { Aire spécifique } \\
\mathrm{m}^{2} \mathrm{~g}^{-1}\end{array}$ \\
\hline Tuile d'argile & 36,56 & 1,05 & $0,2-1,4$ & 3,0 \\
\hline Béton & 14,06 & 0,164 & $0,01-2$ & 6,9 \\
\hline
\end{tabular}

principalement constituées d'aluminosilicates (diopside, anorthite) et de silicates (wollastonite, quartz), et le béton de calcite. Suite à quelques décennies d'existence en milieu urbain, ces matériaux présentent une surface dégradée et polluée. Les tuiles possèdent notamment un dépôt surfacique composé essentiellement de matières organiques, de gypse et de silicates. La porosité totale, l'étendue du domaine des pores et l'aire spécifique de ces matériaux sont données dans le tableau I.

Des tuiles et de grandes plaques de béton ont été disposées dans des bacs de $1 \mathrm{~m}$ de long sur $0,5 \mathrm{~m}$ de large. Ces bacs, d'une superficie de $0,5 \mathrm{~m}^{2}$ ont ensuite été contaminés par dépôt sec d'aérosols pluriélémentaires radioactifs, à l'aide du dispositif POLYR (Pioch and Madoz-Escande, 1995 ; Maubert and Drouet, 1992). Cette contamination est constituée de 180 à $344 \mu \mathrm{g} \mathrm{m}{ }^{-2}$ de césium (stable $+{ }^{134} \mathrm{Cs}$ ), et de 210 à $508 \mu \mathrm{g} \mathrm{m}^{-2}$ de strontium (stable $+{ }^{85} \mathrm{Sr}$ ). La valeur du dépôt surfacique est obtenue par mesure d'une série de membranes réparties autour des surfaces.

\subsection{Les aérosols produits par le dispositif POLYR}

Le dispositif POLYR permet de produire à échelle réduite des aérosols représentatifs de ceux qui seraient émis dans l'atmosphère en cas de fusion partielle du cœur d'un réacteur à eau sous pression de 900 MWe (Pioch and Madoz-Escande, 1995; Maubert and Drouet, 1992).

Leur granulométrie est inférieure à $2 \mu \mathrm{m}$, avec une majorité de particules inférieure à $0,5 \mu \mathrm{m}$. Ils sont constitués le plus souvent de particules cristallisées incluant plusieurs formes métalliques oxydées ou non (principalement Ag et/ou Fe et/ou In et/ou $\mathrm{Cr}$ ). Si le strontium se retrouve dans ces associations métalliques, le césium lui a été détecté dans des particules sphériques associant $\mathrm{Cs}, \mathrm{Cd}$ et I. Une partie de ces éléments difficilement quantifiable doit également se trouver sous forme amorphe (Camarasa-Claret et al., 1997).

En ce qui concerne la dissolution des aérosols, nous avons déjà montré qu'elle n'était pas immédiate (Camarasa-Claret et al., 1997) et que les éléments césium et strontium étaient fortement liés à la présence d'oxydes métalliques. Leur solubilisation directe dans de l'eau de pluie après un temps de contact de $2 \mathrm{~h}$ atteint $80 \%$ pour le césium 
et $70 \%$ pour le strontium. Lorsqu'elle est réalisée en présence de matériaux urbains sur lesquels les aérosols ont été préalablement déposés, la mise en solution est beaucoup plus faible. Pour la tuile, après un temps de contact de $2 \mathrm{~h}$, la mise en solution dans de l'eau de pluie atteint 2-3\% pour le césium et $22-24 \%$ pour le strontium alors qu'elle atteint $12-14 \%$ pour le césium et $21-22 \%$ pour le strontium déposés sur le béton amorphe.

De même nous avons montré que dans des solutions ammoniacales concentrées, la solubilisation est plus importante. Pour la tuile, après un temps de contact de $2 \mathrm{~h}$, la mise en solution dans $\mathrm{NH}_{4} \mathrm{Cl}$ à $0,1 \mathrm{~mol} \mathrm{l}^{-1}$ atteint $41-55 \%$ pour le césium et $32-39 \%$ pour le strontium. Elle atteint $53-67 \%$ pour le césium et $37-42 \%$ pour le strontium déposés sur le béton amorphe (Camarasa-Claret et al., 1997).

\subsection{Protocoles expérimentaux}

Les bacs de $0,5 \mathrm{~m}^{2}$ contenant les surfaces de matériaux urbains sont placés dans un simulateur de pluie à gicleur avec une pente de $5^{\circ}$ pour le béton et de $30^{\circ}$ pour les tuiles.

L'arrosage des bacs (pluie ou lavage) est assuré par une pompe qui alimente une buse de pulvérisation à jet plat. Le gicleur est fixé sur un chariot qui subit un déplacement linéaire de va-et-vient à vitesse constante, perpendiculairement au jet, de façon à balayer entièrement les bacs de $0,5 \mathrm{~m}^{2}$ dans le sens de l'écoulement ( $1 \mathrm{~m})$. Ce système d'aspersion est situé à $2 \mathrm{~m}$ au-dessus de la surface des matériaux contaminés.

Les solutions ruisselant sur les surfaces urbaines sont collectées de façon échelonnée dans le temps en fonction du volume écoulé, puis filtrées à $0,45 \mu \mathrm{m}$. Les filtrats et les filtres sont analysés en spectrométrie $\gamma$, permettant ainsi d'évaluer les fractions désorbées et de différencier les quantités de césium et de strontium éliminées sous forme dissoute ou sous forme particulaire.

L'eau de pluie utilisée est une eau de source, l'eau de Mont Roucous, dont les caractéristiques chimiques sont voisines de celles des eaux de pluie en France. Les solutions de lavage sont composées d'eau de Mont Roucous à laquelle un sel d'ammonium a été rajouté à forte concentration : $\mathrm{NH}_{4} \mathrm{Cl}$ à 0,1 et $1 \mathrm{~mol} \mathrm{l}^{-1} \mathrm{ou}\left(\mathrm{NH}_{4}\right)_{2} \mathrm{C}_{2} \mathrm{O}_{4}$ à $0,1 \mathrm{~mol} \mathrm{l}^{-1}$. Ce cation a été choisi compte tenu de ses propriétés légèrement acides et de son caractère faiblement hydraté qui lui permet de s'échanger facilement.

Cinq procédures de ruissellement sur les surfaces urbaines ont été reproduites expérimentalement (Tab. II). Les procédures 1 à 4 comprennent 3 séquences de pluies suivies, après un temps de séchage, de lavages avec l'ion ammonium sous différentes formes ; la procédure 5 examine l'influence des seuls lavages sans pluies préalables. 
IMPACT DE QUELQUES TECHNIQUES DE LAVAGES..

TABLEAU II

Procédures expérimentales lors des essais de ruissellement.

Experimental procedures during the tests of runoff.

\begin{tabular}{|c|c|c|c|c|c|c|}
\hline \multirow{2}{*}{ Procédure } & \multicolumn{2}{|r|}{ Pluie } & \multirow{2}{*}{$\begin{array}{l}\text { Arrêt entre } \\
\text { pluie et lavage }\end{array}$} & \multicolumn{3}{|c|}{ Lavage } \\
\hline & $\begin{array}{c}\text { intensité } \\
\mathrm{mm} \mathrm{h}^{-1}\end{array}$ & séquence & & solution & $\begin{array}{c}\text { débit } \\
1 \mathrm{~h}^{-1} \mathrm{~m}^{-2}\end{array}$ & séquence \\
\hline 1 & 10 & $\begin{array}{l}3 \text { pluies de } 1 \mathrm{~h} \text {, arrêt } \\
\text { entre les pluies: } \\
23 \mathrm{~h}\end{array}$ & $8 \mathbf{j}$ & $\begin{array}{c}\mathrm{NH}_{4} \mathrm{Cl} \\
0,1 \mathrm{~mol} \mathrm{l}^{-1}\end{array}$ & 5 & $\begin{array}{l}2 \text { lavages de } 20 \mathrm{~min} \\
\text { et un de } 30 \mathrm{~min} \text {, } \\
\text { arrêt entre les } \\
\text { lavages : } 25 \mathrm{~h} \text { et } 4 \mathrm{~h} \text {. }\end{array}$ \\
\hline 2 & 10 & $\left|\begin{array}{l}3 \text { pluies de } 1 \mathrm{~h} \text {, arrêt } \\
\text { entre les pluies : } \\
23 \mathrm{~h}\end{array}\right|$ & $11 \mathrm{j}$ & $\begin{array}{l}\left(\mathrm{NH}_{4}\right)_{2} \mathrm{C}_{2} \mathrm{O}_{4} \\
0,1 \mathrm{~mol} \mathrm{l}^{-1}\end{array}$ & 5 & $\begin{array}{l}2 \text { lavages de } 20 \mathrm{~min} \\
\text { et un de } 30 \mathrm{~min} \text {, } \\
\text { arrêt entre les } \\
\text { lavages: } 3 \mathrm{~h} \text { et } 2 \mathrm{~h} \text {. }\end{array}$ \\
\hline 3 & 5 & $\begin{array}{l}3 \text { pluies de } 1 \mathrm{~h} \text {, arrêt } \\
\text { entre les pluies : } \\
23 \mathrm{~h}\end{array}$ & $9 \mathrm{j}$ & $\begin{array}{c}\mathrm{NH}_{4} \mathrm{Cl} \\
0,1 \mathrm{~mol} \mathrm{l}^{-1}\end{array}$ & 5 & $\begin{array}{l}2 \text { lavages de } 30 \mathrm{~min} \\
\text { et un de } 45 \mathrm{~min} \text {, } \\
\text { arrêt entre les } \\
\text { lavages: } 3 \mathrm{~h} \text { et } 1 \mathrm{~h} 30 \text {. }\end{array}$ \\
\hline 4 & 5 & $\begin{array}{l}3 \text { pluies de } 1 \mathrm{~h} \text {, arrêt } \\
\text { entre les pluies : } \\
23 \mathrm{~h}\end{array}$ & $14 \mathrm{j}$ & $\begin{array}{c}\mathrm{NH}_{4} \mathrm{Cl} \\
1 \mathrm{~mol}^{-1}\end{array}$ & 5 & $\begin{array}{l}2 \text { lavages de } 30 \mathrm{~min} \\
\text { et un de } 45 \mathrm{~min} \text {, } \\
\text { arrêt entre les } \\
\text { lavages: } 2 \mathrm{~h} \text { et } 3 \mathrm{~h} 30 \text {. }\end{array}$ \\
\hline 5 & & & & $\begin{array}{c}\mathrm{NH}_{4} \mathrm{Cl} \\
0,1 \mathrm{~mol} \mathrm{l}^{-1}\end{array}$ & 5 & $\begin{array}{l}3 \text { lavages de } 30 \mathrm{~min} \\
\text { et un de } 60 \mathrm{~min} \text {, } \\
\text { arrêt entre les } \\
\text { lavages: } 1 \mathrm{~h} 30,2 \mathrm{~h} 30 \\
\text { et } 1 \mathrm{~h} 30 \text {. }\end{array}$ \\
\hline
\end{tabular}

\section{Résultats et discussion}

\subsection{Décontamination naturelle par l'eau de pluie}

Après $3 \mathrm{~h}$ de pluie, la rétention des radionucléides sur les matériaux urbains reste très élevée pour les deux intensités étudiées. La fraction résiduelle en césium (Fig. 1) est de $0,98-0,99$ sur la tuile et de $0,94-0,95$ pour le béton. Pour les deux matériaux la décontamination du strontium est meilleure que celle du césium et semble augmenter lorsque l'intensité de la pluie diminue (fraction résiduelle du strontium sur la tuile $0,845 \pm 0,015$ pour une intensité de $5 \mathrm{~mm} \mathrm{~h}^{-1}$ et $0,91 \pm 0,01$ pour $10 \mathrm{~mm} \mathrm{~h}^{-1}$. 
C. CAMARASA-CLARET et al.

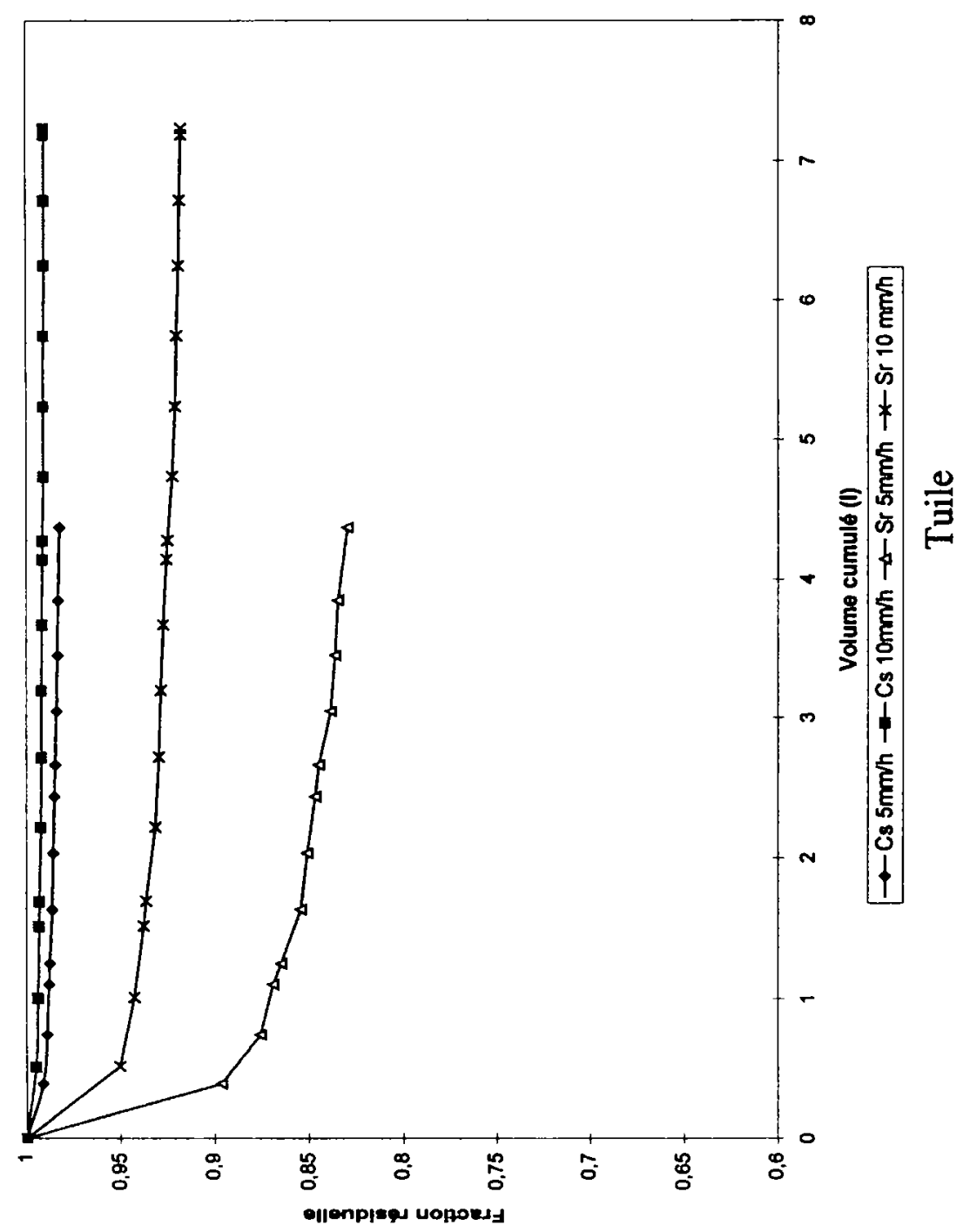


IMPACT DE QUELQUES TECHNIQUES DE LAVAGES...
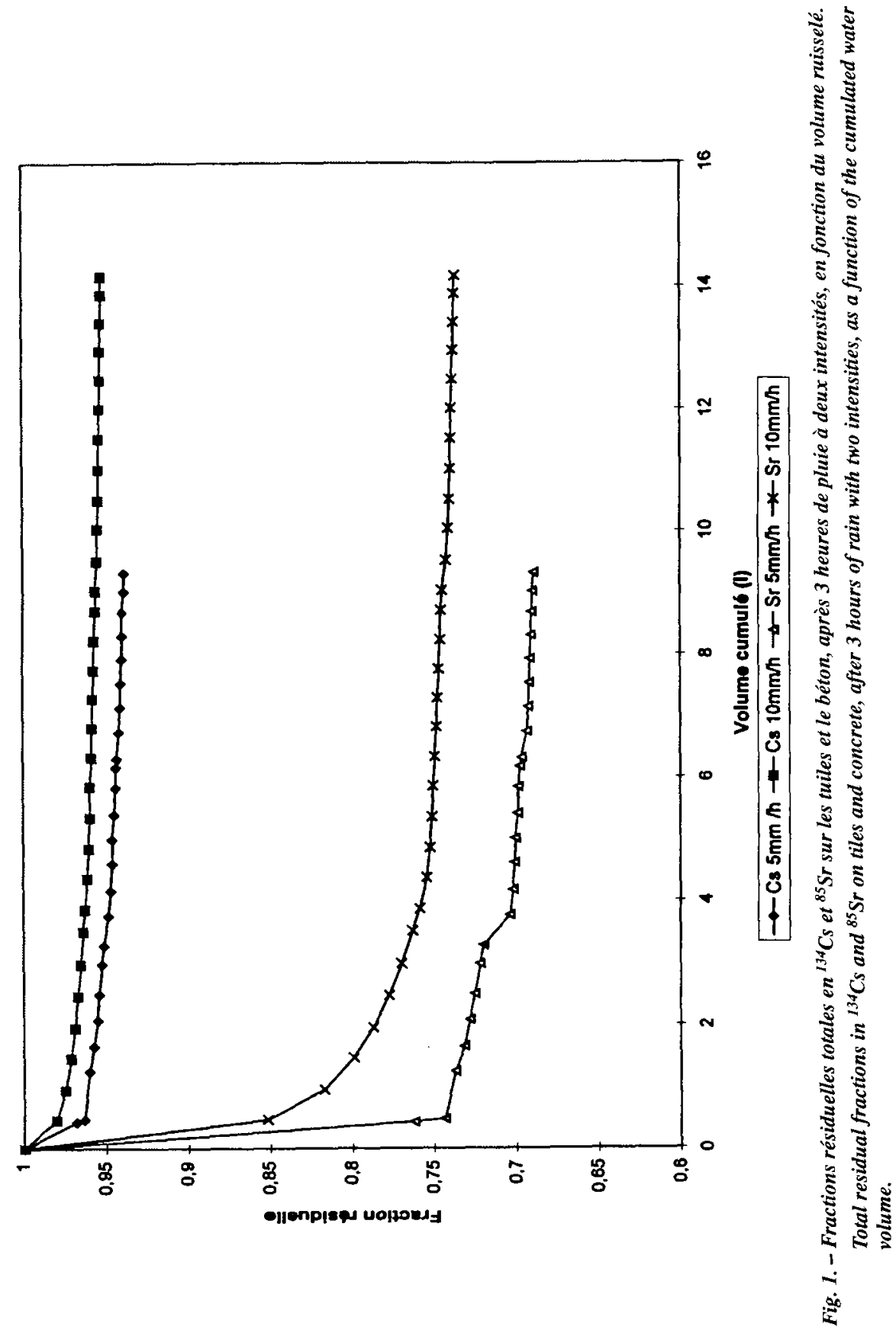

RADIOPROTECTION - VOL. 35 - $\mathrm{N}^{\circ} 1$ (2000) 
Enfin la contamination résiduelle du béton est plus faible que celle de la tuile (fraction résiduelle en strontium du béton $0,68 \pm 0,01$ pour une intensité de $5 \mathrm{~mm} \mathrm{~h}^{-1}$ et $0,72 \pm 0,01$ pour $10 \mathrm{~mm} \mathrm{~h}^{-1}$ ). Ce dernier point peut en partie s'expliquer par une pénétration plus importante des aérosols dans la tuile due à sa plus grande porosité. En effet, le diamètre moyen des pores des tuiles déterminé par porosimétrie
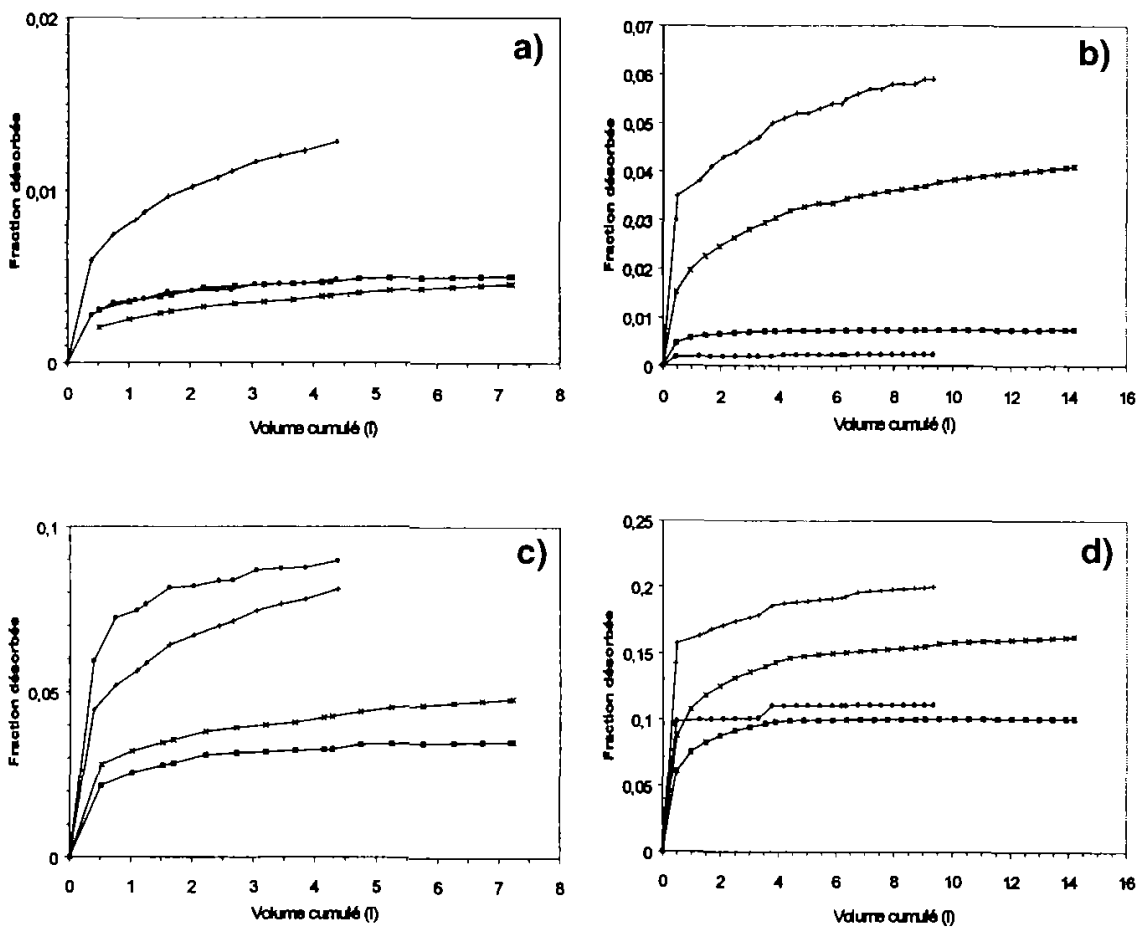

\begin{tabular}{|l|l|}
\hline$\longleftarrow$ Dissous- pluie $5 \mathrm{~mm} \cdot \mathrm{h}^{-1}$ & $\star-\quad$ Dissous- pluie $10 \mathrm{~mm} \cdot \mathrm{h}^{-1}$ \\
\hline$\longleftarrow \quad$ Particulaire - pluie $5 \mathrm{~mm} \cdot \mathrm{h}^{-1}$ & $-\quad$ Particulaire - pluie $10 \mathrm{~mm} \cdot \mathrm{h}^{-1}$ \\
\hline
\end{tabular}

Fig. 2. - Fractions désorbées en ${ }^{134} \mathrm{Cs}$ et en ${ }^{85} \mathrm{Sr}$ dissous et particulaires, obtenues après 3 heures de pluie à deux intensités, sur la tuile en argile et sur le béton, en fonction du volume ruisselé. a) tuile d'argile - $C s, b)$ béton - $C s, c)$ tuile d'argile $-S r, d)$ béton $-S r$.

Dissolved and particular desorbed fractions of ${ }^{134} \mathrm{Cs}$ and ${ }^{85} \mathrm{Sr}$ after 3 hours of rain at two intensities, over clay tile and cement versus the cumulated water volume.

a) tiles - Cs, b) concrete - Cs, c) tiles - Sr, d) concrete - Sr. 
au mercure est de 1,05 $\mu \mathrm{m}$ alors que celui du béton est de $0,16 \mu \mathrm{m}$. Après entraînement mécanique par l'eau, ceux-ci se trouveraient piégés dans les pores de ce matériau rendant leur dissolution plus difficile.

L'évolution de la fraction désorbée sous forme dissoute et sous forme particulaire en fonction du volume ruisselé (Fig. 2) montre que plus l'intensité de la pluie est faible, plus la remobilisation des radionucléides est importante, sauf dans le cas du césium éliminé du béton sous forme particulaire. L'élimination plus importante à faible intensité avait déjà été mise en évidence par Pioch and Madoz-Escande (1995), lors de pluies à $10 \mathrm{~mm} \mathrm{~h}^{-1}$ et $30 \mathrm{~mm} \mathrm{~h}^{-1}$ sur divers matériaux urbains neufs. Il apparaît donc que le paramètre «temps de contact» de l'eau sur le matériau influence davantage les processus de décontamination que les paramètres «volume de la lame d'eau » et «vitesse d'écoulement du fluide». Ce dernier paramètre, qui joue un rôle lors de l'entraînement des particules, est par contre plus important dans le cas de la remobilisation particulaire du césium sur le béton.

On observe dans le cas du strontium particulaire des points singuliers au niveau des interruptions et reprises de la pluie qui correspondent sur le graphique aux ruptures de pente. Le gradient de pression de l'eau dans les pores très élevé en début de pluie permet d'extraire et d'entraîner une partie plus importante d'aérosols non encore dissous. Le développement ultérieur de la zone mouillée par diffusion de l'eau sur les surfaces inhibe cet effet d'entraînement, l'impact des gouttes d'eau et leur effet d'arrachement étant plus faibles. Pendant les phases d'arrêt, il se produit une dissolution des aérosols dans la pellicule d'eau superficielle, puis un assèchement de la surface. Lors d'une nouvelle pluie, le même phénomène agressif se reproduit entraînant une augmentation brusque des fractions dissoutes et particulaires, qui est minimisé ensuite. Ce phénomène apparaît peu ou pas dans le cas du césium déposé sur le béton, le césium dissous se sorbant sur le béton lors des phases d'arrêt (forte affinité du césium avec le gel CSH du béton, Camarasa-Claret et al. 1997).

La forme de ces courbes montre que c'est le début de la première pluie qui provoque l'essentiel de la remobilisation des radionucléides, aussi bien sous forme dissoute que particulaire. Les pluies suivantes sont plus faiblement décontaminantes. Une fois le matériau mouillé, l'élimination des radionucléides est très faible, comme le constataient Pioch and Madoz-Escande (1995) et Roed (1987).

\subsection{Décontamination artificielle}

\subsubsection{Influence de la nature de la solution ammoniacale}

Les résultats concernant les lavages pratiqués après une séquence pluvieuse (procédures 1 à 4) sont présentés dans la figure 3. Quelle que soit la procédure appliquée, la contamination résiduelle reste élevée. La comparaison des valeurs obtenues après les procédures 1 et 2 (effet du chlorure ou de l'oxalate d'ammonium), 


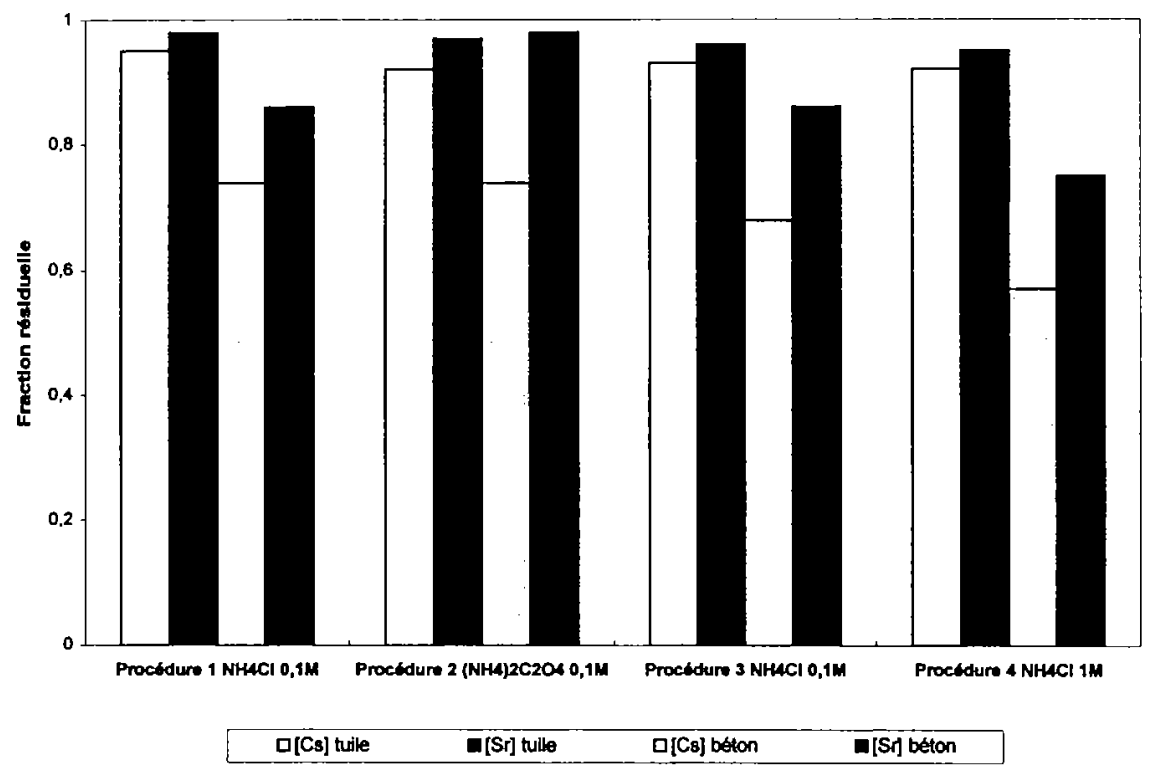

Fig. 3. - Fractions résiduelles en Cs et en Sr des tuiles et du béton après différentes procédures de lavage pratiquées suite à une séquence pluvieuse (voir protocoles expérimentaux).

Cs and Sr Residual fractions on cement and clay tile after various procedures of washing subsequent to a rain-water sequence.

ne montre pas de différence significative dans l'efficacité du lavage de la tuile, aussi bien pour le césium que pour le strontium. Pour le béton, les valeurs de décontamination en césium sont un peu meilleures, mais sans différence pour les deux procédures. Par contre, alors qu'il est en partie désorbé par la solution de $\mathrm{NH}_{4} \mathrm{Cl}$, le strontium reste en quasi-totalité sur le béton lors du lavage avec la solution de $\left(\mathrm{NH}_{4}\right)_{2} \mathrm{C}_{2} \mathrm{O}_{4}$. L'effet complexant de l'oxalate n'améliore pas la remobilisation des radionucléides contrairement aux résultats que nous avions obtenus lors des essais en flacons. En présence de cet ion, la décontamination du strontium devient même quasiment nulle par coprécipitation avec les ions carbonate libérés lors de la formation d'oxalate de calcium. Une solution de chlorure d'ammonium est donc préférable à celle d'oxalate car elle induit beaucoup moins de phénomènes de précipitation.

La comparaison des valeurs obtenues lors des procédures 3 et 4 montre que l'élimination du césium et du strontium est plus importante lorsque la concentration en $\mathrm{NH}_{4} \mathrm{Cl}$ de la solution de lavage est plus élevée. Cependant l'utilisation pratique d'une telle solution demeure douteuse compte tenu de son coût et de ses effets corrosifs. 


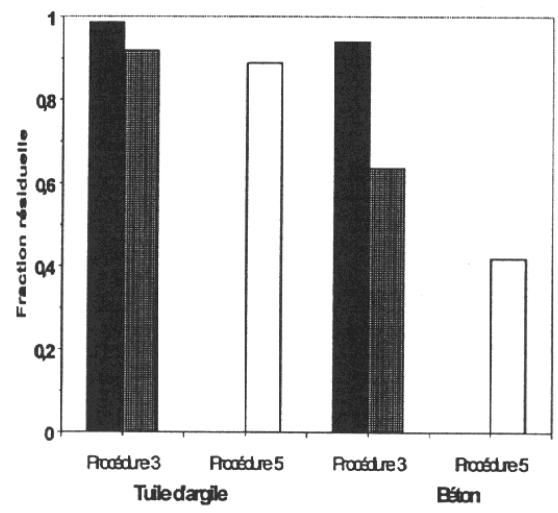

Césium

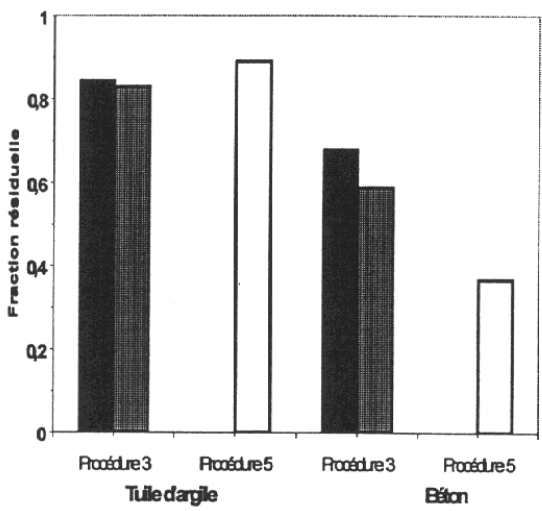

Strontium

\begin{tabular}{|l|l|l|l|}
\hline pluie & pluie+lavage $\square$ lavage \\
\end{tabular}

Fig. 4. - Fractions résiduelles en ${ }^{134} \mathrm{Cs}$ et en ${ }^{85} \mathrm{Sr}$, obtenues après lavage avec $\mathrm{NH}_{4} \mathrm{Cl} 0,1 \mathrm{M}$ : suite à une pluie de $5 \mathrm{~mm} \mathrm{~h}^{-1}$ pendant 3 heures (procédure 3), sans pluie préalable (procédure 5).

${ }^{134} \mathrm{Cs}$ and ${ }^{85} \mathrm{Sr}$ residual fractions after washing with $\mathrm{NH}_{4} \mathrm{Cl} 0,1 \mathrm{M}$ : after a rain of $5 \mathrm{mmh}^{-1}$ during 3 hours (procedure 3), without preliminary rain (procedure 5).

\subsubsection{Influence de la procédure d'aspersion}

Les fractions résiduelles en césium et strontium résultant de l'application des procédures 3 et 5 sur la tuile et le béton sont présentées sur la figure 4 .

On constate que quel que soit le lavage, la remobilisation des radionucléides est meilleure lorsqu'il n'y a pas eu de pluie entre le dépôt d'aérosols et ce lavage. Le lavage direct est alors plus efficace que lorsqu'il est précédé d'une pluie, sauf pour le strontium déposé sur la tuile d'argile. Ceci est en accord avec les conclusions de Sinnaeve et Olast (1991), qui signalaient que l'efficacité du lavage décroissait rapidement avec l'âge de la contamination.

Toutefois, la contamination résiduelle sur les matériaux urbains reste élevée, de l'ordre de $90 \%$ sur la tuile et de $40 \%$ sur le béton.

\section{Conclusion}

L'efficacité décontaminante de pluies et de lavages sur des matériaux préalablement contaminés par du césium et du strontium radioactifs a été évaluée au cours d'expérimentations en milieu contrôlé. 
L'eau de pluie présente un faible pouvoir de remobilisation des radionucléides. Après $3 \mathrm{~h}$ de pluie à l'intensité de $5 \mathrm{~mm} \mathrm{~h}^{-1}$, la contamination résiduelle en césium demeure élevée: $98-99 \%$ sur la tuile d'argile et 93-94\% sur le béton. Pour le strontium, elle s'élève à $83-86 \%$ sur la tuile et $67-69 \%$ sur le béton. L'élimination intervient essentiellement en début de la première pluie et la décontamination naturelle est plus efficace lorsque l'intensité de la pluie est faible. La pluie ne permettra donc en aucun cas de décontaminer significativement des matériaux urbains. Ceci vient confirmer les valeurs trouvées dans la littérature : environ un an après l'accident de Tchernobyl, les fractions résiduelles en césium sont proches ou supérieures à $80 \%$ sur des tuiles, et à $70 \%$ sur du béton (Sandalls, 1985 ; Roed, 1987 ; Nicholson, 1988; Wilkins 1987).

La décontamination artificielle par aspersion de solutions ammoniacales concentrées est plus efficace que la décontamination naturelle due à la pluie car ces solutions ont un caractère échangeur vis-à-vis de la tuile et agressif vis-à-vis du béton. La contamination résiduelle la plus faible est obtenue après $2 \mathrm{~h} 30 \mathrm{~min}$ de lavage direct sans pluie préalable avec la solution $\mathrm{NH}_{4} \mathrm{Cl}$ à 0,1 mole $\mathrm{I}^{-1}$, au débit de $51 \mathrm{~h}^{-1} \mathrm{~m}^{-2}$. Environ $90 \%$ des radionucléides étudiés demeurent sur la tuile et $40 \%$ sur le béton. L'efficacité de cette technique diminue au moins de moitié s'il y a une pluie entre la contamination et le lavage.

Ces résultats confirment, pour des formes physico-chimiques particulaires et des conditions expérimentales représentatives d'un rejet accidentel, la difficulté de déplacer par voie aqueuse cette contamination. Les mécanismes mis en jeu lors de ce déplacement sont ici abordés et permettent de mieux comprendre les raisons de ce faible transfert. Nous estimons que les taux de décontamination obtenus ne sont pas suffisamment significatifs, même si l'élimination sur le béton n'est pas négligeable, pour justifier que l'on passe à une étape ultérieure qui consisterait à réaliser une étude de faisabilité opérationnelle.

\section{RÉFÉRENCES}

Arnaud A. (1995) Réhabilitation des sols et surfaces après accident : contribution à l'étude de la décontamination en césium et strontium par des polyacrylamides fonctionnalisés. Thèse spécialité chimie de l'environnement et santé. Université Aix-Marseille. 195p + annexes.

Camarasa-Claret C., Persin F., Real J. (1997) Dissolution du césium et du strontium contenus dans des aérosols radioactifs accidentels. Actes du $13^{e}$ congrès français sur les aérosols, 2-3 décembre Paris, pp. 25-31.

Devell L. (1989) Characteristics of the Chernobyl release and fallout that affect the transport and behaviour of radioactive substances in the environment. Recent advances in reactor accident consequences assessment, Rome (Italy).

De Witt H., Goldammer W., Brenk H.D., Hille R., Jacobs H. (1990) Decontamination stratégies in urban areas after nuclear accidents. Seminar on methods and codes for assessing the off-site conséquences of nuclear accident. 7-11 may, Athens, pp. 615-632.

Gauchon J.P., Brunel G., Costes J. R., Faucompré B. (1991) Mousse de décontamination à.durée de vie contrôlée et installation de décontamination d'objets utilisant une telle mousse. Commisșariat à l'énergie atomique, $\mathrm{n}^{\circ} 9109286,-07 / 91$. 
IMPACT DE QUELQUES TECHNIQUES DE LAVAGES ..

Maubert H., Drouet P. (1992) Chemical speciation of radionuclides. In: Evaluation of data on the transfer of radionuclides in the food chain, (G. Desmet, J. Sinnaeve, Eds.), pp. 3-48.

Nicholson KW (1987) Deposition of caesium to surfaces of buildings. Radiat. Prot. Dosim. 21 (1/3), $37-42$.

Nicholson K.W. (1988) The rétention of ${ }^{134} \mathrm{Cs}$ and ${ }^{137} \mathrm{Cs}$ by roof tiles. In Nuclear Science and Technology, (M. Olast, J. Sinnaeve, Eds.): CEC Brussels, EUR-11408 pp. 193-199.

Nicholson K.W., Hedgecock J.B. (1991) Behavior of radioactivity from Chernobyl - Weathering from buildings. J. Environ Radioactivity 14, 225-231.

Pioch M., Madoz-Escande C. (1995) Effect of rainwater on the remobilisation and dissolution of $134 \mathrm{Cs}$ and $85 \mathrm{Sr}$ contained in aerosols similar to those discharged in a nuclear accident and deposited in an urban environment. J. Environ. Radioactivity 26, 51-61.

Roed J. (1987) Run-off from and weathering of roof material following the Chernobyl accident. Radiat. Prot. Dosim. 21, 59-63.

Roed J., Sandalls J. (1989) The concentration levels of Chernobyl fallout on different surfaces in Gävle in Sweden. In: Proceedings of the XVth regional congress of IRPA, September 1989, Visby, Sweden, pp. $367-371$.

Roed J., Jacob J. (1990) Deposition on urban surfaces and subsequent weathering. In: Seminar on methods and codes for assessing the off-site conséquences of nuclear accident, 7-11 may 1990, Athens, pp. 335-356.

Roed J., Andersson K.G. (1996) Clean-up of urban areas in the CIS countries contamined by Chernobyl fallout. J. Environ. Radioactivity 33, 107-116.

Sandalls F.J., Stewart S.P., Wilkins B.T. ( 1986) Natural and forced décontamination of urban surfaces contaminated with radiocaesium. In: Proceedings in the workshop on methods for assessing the off-site radiological conséquences of nuclear accidents, 15-19 April 1985, Luxembourg, pp. 51 1-532.

Sinnaeve J., Olast M. (1991) Improvement of practical counter measures: the urban environment - PostChernobyl action. Commission of the Europan Communities, Publisher Luxembourg, EUR 12555, 311.

Wilkins B.T. (1987) The retention behavior of radiocesium on common building materials under naturel outdoor conditions. Radiat. Prot. Dosim. 21, 69-73. 